\title{
New Ostrowski type inequalities for harmonically convex functions
}

\author{
Tuncay Koroglu \\ Karadeniz Technical University, Department of Mathemaics, Trabzon, Turkey \\ Received: 11 December 2017, Accepted: 15 December 2017 \\ Published online: 7 March 2018.
}

Abstract: In this work, we pose a new equality for differentiable functions. By using this equality, we have some new Ostrowki type inequalities and some error estimates for the midpoint formula for functions whose derivatives in absolute values at certain powers are harmonically convex.

Keywords: Ostrowski type inequalities, midpoint type inequalities, harmonically convex function.

\section{Introduction}

The following result is known in the literature as Ostrowski's inequality [7];

Theorem 1. [2,8]. Let $f:[a, b] \rightarrow \mathbb{R}$ be a differentiable mapping on $(a, b)$ with the property that $\left|f^{\prime}(t)\right| \leq M$ for all $t \in(a, b)$. Then

$$
\left|f(x)-\frac{1}{b-a} \int_{a}^{b} f(t) d t\right| \leq(b-a) M\left[\frac{1}{4}+\frac{\left(x-\frac{a+b}{2}\right)^{2}}{(b-a)^{2}}\right]
$$

for all $x \in[a, b]$.

The constant $\frac{1}{4}$ is the best possible it means that it cannot be replaced by a smaller constant.

The inequality (1) can be expressed in the following form:

$$
\left|f(x)-\frac{1}{b-a} \int_{a}^{b} f(t) d t\right| \leq \frac{M}{b-a}\left[\frac{(x-a)^{2}+(x-b)^{2}}{2}\right]
$$

For some results which generalize, improve and extend the inequalities (1) and (2) we refer the reader to the recent papers (see $[1,2,4,5,7,8,9])$.

In [3], İşcan gave definition of harmonically convex functions and Hermite-Hadamard type inequality for harmonically convex functions as follows:

Definition 1. [3]. Let $I \subset \mathbb{R} \backslash\{0\}$ be a real interval. A function $f: I \rightarrow \mathbb{R}$ is said to be harmonically convex, if

$$
f\left(\frac{x y}{t x+(1-t) y}\right) \leq t f(y)+(1-t) f(x)
$$

\footnotetext{
${ }^{*}$ Corresponding author e-mail: tkor@ktu.edu.tr
} 
for all $x, y \in I$ and $t \in[0,1]$. If the inequality in (3) is reversed, then $f$ is said to be harmonically concave.

Theorem 2. [3]. Let $f: I \subset \mathbb{R} \backslash\{0\} \rightarrow \mathbb{R}$ be a harmonically convex function and $a, b \in I$ with $a<b$. If $f \in L[a, b]$ then the following inequalities holds:

$$
f\left(\frac{2 a b}{a+b}\right) \leq \frac{a b}{b-a} \int_{a}^{b} \frac{f(x)}{x^{2}} d x \leq \frac{f(a)+f(b)}{2} .
$$

We will use the following special functions called beta function and hypergeometric function, respectively in the literature

$$
\begin{gathered}
\beta(x, y)=\frac{\Gamma(x) \Gamma(y)}{\Gamma(x+y)}=\int_{0}^{1} t^{x-1}(1-t)^{y-1} d t, x, y>0, \\
{ }_{2} F_{1}(a, b ; c ; z)=\frac{1}{\beta(b, c-b)} \int_{0}^{1} t^{b-1}(1-t)^{c-b-1}(1-z t)^{-a} d t,
\end{gathered}
$$

where $c>b>0$, and $|z|<1$ (see e.g. [6]).

\section{New Ostorowski type inequalities}

The following lemma is important to prove our main results.

Lemma 1.. Let $f: I \subset \mathbb{R} \backslash\{0\} \rightarrow \mathbb{R}$ be a differentiable function on $I^{\circ}$ such that $a, b \in I$ with $a<b$. If $f^{\prime} \in L[a, b]$ then

$$
f(x)-\frac{a b}{b-a} \int_{a}^{b} \frac{f(u)}{u^{2}} d u=a b(b-a) \int_{0}^{1} \frac{p(t)}{(t a+(1-t) b)^{2}} f^{\prime}\left(\frac{a b}{t a+(1-t) b}\right) d t
$$

where

$$
p(t)= \begin{cases}t, & t \in\left[0, \frac{(x-a) b}{(b-a) x}\right] \\ t-1, & t \in\left(\frac{(x-a) b}{(b-a) x}, 1\right]\end{cases}
$$

for all $x \in[a, b]$.

Proof.

$$
\begin{aligned}
& a b(b-a) \int_{0}^{1} \frac{p(t)}{(t a+(1-t) b)^{2}} f^{\prime}\left(\frac{a b}{t a+(1-t) b}\right) d t=a b(b-a)\left[\int_{0}^{\frac{(x-a) b}{(b-a) x}} \frac{t}{(t a+(1-t) b)^{2}} f^{\prime}\left(\frac{a b}{t a+(1-t) b}\right) d t\right. \\
& \left.\quad+\int_{\frac{(x-a) b}{(b-a) x}}^{1} \frac{t-1}{(t a+(1-t) b)^{2}} f^{\prime}\left(\frac{a b}{t a+(1-t) b}\right) d t\right] \\
& =\left[\left.t f\left(\frac{a b}{t a+(1-t) b}\right)\right|_{0} ^{\frac{(x-a) b}{(b-a) x}}-\int_{0}^{\frac{(x-a) b}{(b-a) x}} f\left(\frac{a b}{t a+(1-t) b}\right) d t+\left.(t-1) f\left(\frac{a b}{t a+(1-t) b}\right)\right|_{\frac{(x-a) b}{(b-a) x}} ^{1}-\int_{\frac{(x-a) b}{(b-a) x}}^{1} f\left(\frac{a b}{t a+(1-t) b}\right) d t\right] \\
& =\left[\frac{(x-a) b}{(b-a) x} f(x)-\frac{a b}{b-a} \int_{a}^{x} \frac{f(u)}{u^{2}} d u+\frac{(b-x) a}{(b-a) x} f(x)-\frac{a b}{b-a} \int_{x}^{b} \frac{f(u)}{u^{2}} d u\right] \\
& =f(x)-\frac{a b}{b-a} \int_{a}^{b} \frac{f(u)}{u^{2}} d u .
\end{aligned}
$$

This completes the proof. 
Theorem 3. Let $f: I \subset(0, \infty) \rightarrow \mathbb{R}$ be a differentiable function on $I^{\circ}$ such that $a, b \in I$ with $a<b$ and $f^{\prime} \in L$. If $\left|f^{\prime}\right|$ is harmonically convex on $[a, b]$, then for all $x \in[a, b]$, we have

$$
\left|f(x)-\frac{a b}{b-a} \int_{a}^{b} \frac{f(u)}{u^{2}} d u\right| \leq a b(b-a)\left[\begin{array}{c}
\left|f^{\prime}(a)\right|\left(T_{1}(a, b, x)+T_{3}(a, b, x)\right) \\
+\left|f^{\prime}(b)\right|\left(T_{2}(a, b, x)+T_{4}(a, b, x)\right)
\end{array}\right]
$$

where

$$
\begin{gathered}
T_{1}(a, b, x)=\left[\begin{array}{c}
\frac{1}{2}\left(\frac{(x-a)}{(b-a) x}\right)_{2}^{2}{ }_{2} F_{1}\left(2,2 ; 3 ; \frac{(x-a) b}{(b-a) x}\left(1-\frac{a}{b}\right)\right) \\
-\frac{b}{3}\left(\frac{(x-a)}{(b-a) x}\right)_{2}^{3}{ }_{2} F_{1}\left(2,3 ; 4 ; \frac{(x-a) b}{(b-a) x}\left(1-\frac{a}{b}\right)\right)
\end{array}\right], \\
T_{2}(a, b, x)=\frac{b}{3}\left(\frac{(x-a)}{(b-a) x}\right)^{3}{ }_{2} F_{1}\left(2,3 ; 4 ; \frac{(x-a) b}{(b-a) x}\left(1-\frac{a}{b}\right)\right), \\
T_{3}(a, b, x)=\left[\begin{array}{c}
-\frac{(x-a) b^{-1}}{(b-a) x}{ }_{2} F_{1}\left(2,1 ; 2 ; \frac{(x-a) b}{(b-a) x}\left(1-\frac{a}{b}\right)\right) \\
+\left(\frac{(x-a)}{(b-a) x}\right)_{2}{ }_{2} F_{1}\left(2,2 ; 3 ; \frac{(x-a) b}{(b-a) x}\left(1-\frac{a}{b}\right)\right) \\
-\frac{b}{3}\left(\frac{(x-a)}{(b-a) x}\right)^{3}{ }_{2} F_{1}\left(2,3 ; 4 ; \frac{(x-a) b}{(b-a) x}\left(1-\frac{a}{b}\right)\right)
\end{array}\right], \\
T_{4}(a, b, x)=\left[\begin{array}{c}
\frac{b^{-2}}{6}{ }_{2} F_{1}\left(2,2 ; 4 ;\left(1-\frac{a}{b}\right)\right) \\
-\frac{1}{2}\left(\frac{(x-a)}{(b-a) x}\right)_{2}{ }_{2} F_{1}\left(2,2 ; 3 ; \frac{(x-a) b}{(b-a) x}\left(1-\frac{a}{b}\right)\right) \\
+\frac{b}{3}\left(\frac{(x-a)}{(b-a) x}\right)_{2}{ }_{2} F_{1}\left(2,3 ; 4 ; \frac{(x-a) b}{(b-a) x}\left(1-\frac{a}{b}\right)\right)
\end{array}\right] .
\end{gathered}
$$

Proof. By using Lemma 1 and harmonically convexity of $\left|f^{\prime}\right|$, we have

$$
\begin{aligned}
\left|f(x)-\frac{a b}{b-a} \int_{a}^{b} \frac{f(u)}{u^{2}} d u\right| & \leq a b(b-a)\left[\int_{0}^{\frac{(x-a) b}{(b-a) x}} \frac{t}{(t a+(1-t) b)^{2}}\left|f^{\prime}\left(\frac{a b}{t a+(1-t) b}\right)\right| d t\right. \\
& \left.+\int_{\frac{(x-a) b}{(b-a) x}}^{1} \frac{1-t}{(t a+(1-t) b)^{2}}\left|f^{\prime}\left(\frac{a b}{t a+(1-t) b}\right)\right| d t\right] \\
& \leq a b(b-a)\left[\int_{0}^{\frac{(x-a) b}{(b-a) x}} \frac{t}{(t a+(1-t) b)^{2}}\left[(1-t)\left|f^{\prime}(a)\right|+t\left|f^{\prime}(b)\right|\right] d t\right. \\
& \left.+\int_{\frac{(x-a) b}{(b-a) x}}^{1} \frac{1-t}{(t a+(1-t) b)^{2}}\left[(1-t)\left|f^{\prime}(a)\right|+t\left|f^{\prime}(b)\right|\right] d t\right] \\
& \leq a b(b-a)\left[\left|f^{\prime}(a)\right| \int_{0}^{\frac{(x-a) b}{(b-a) x}} \frac{t(1-t)}{(t a+(1-t) b)^{2}} d t+\left|f^{\prime}(b)\right| \int_{0}^{\frac{(x-a) b}{(b-a) x}} \frac{t^{2}}{(t a+(1-t) b)^{2}} d t\right. \\
& \left.+\left|f^{\prime}(a)\right| \int_{\frac{(x-a) b}{(b-a) x}}^{1} \frac{(1-t)^{2}}{(t a+(1-t) b)^{2}} d t+\left|f^{\prime}(b)\right| \int_{\frac{(x-a) b}{(b-a) x}}^{1} \frac{t(1-t)}{(t a+(1-t) b)^{2}} d t\right] .
\end{aligned}
$$

If we calculate the appearing integrals with hypergeometric functions, we have 


$$
\begin{aligned}
& \int_{0}^{\frac{(x-a) b}{(b-a) x}} \frac{t(1-t)}{(t a+(1-t) b)^{2}} d t=\int_{0}^{\frac{(x-a) b}{(b-a) x}} \frac{t}{(t a+(1-t) b)^{2}} d t-\int_{0}^{\frac{(x-a) b}{(b-a) x}} \frac{t^{2}}{(t a+(1-t) b)^{2}} d t \\
& =\left(\frac{(x-a)}{(b-a) x}\right)^{2} \int_{0}^{1} u\left(1-\left[\frac{(x-a) b}{(b-a) x}\left(1-\frac{a}{b}\right)\right] u\right)^{-2} d u \\
& -b\left(\frac{(x-a)}{(b-a) x}\right)^{3} \int_{0}^{1} u^{2}\left(1-\left[\frac{(x-a) b}{(b-a) x}\left(1-\frac{a}{b}\right)\right] u\right)^{-2} d u \\
& =\left[\begin{array}{c}
\frac{1}{2}\left(\frac{(x-a)}{(b-a) x}\right)_{2}^{2} F_{1}\left(2,2 ; 3 ; \frac{(x-a) b}{(b-a) x}\left(1-\frac{a}{b}\right)\right) \\
-\frac{b}{3}\left(\frac{(x-a)}{(b-a) x}\right)_{2}^{3} F_{1}\left(2,3 ; 4 ; \frac{(x-a) b}{(b-a) x}\left(1-\frac{a}{b}\right)\right)
\end{array}\right]=T_{1}(a, b, x), \\
& \int_{0}^{\frac{(x-a) b}{(b-a) x}} \frac{t^{2}}{(t a+(1-t) b)^{2}} d t=\frac{b}{3}\left(\frac{(x-a)}{(b-a) x}\right)^{3}{ }_{2} F_{1}\left(2,3 ; 4 ; \frac{(x-a) b}{(b-a) x}\left(1-\frac{a}{b}\right)\right)=T_{2}(a, b, x) \\
& \int_{\frac{(x-a) b}{(b-a) x}}^{1} \frac{(1-t)^{2}}{(t a+(1-t) b)^{2}} d t=\int_{0}^{1} \frac{(1-t)^{2}}{(t a+(1-t) b)^{2}} d t-\int_{0}^{\frac{(x-a) b}{(b-a) x}} \frac{(1-t)^{2}}{(t a+(1-t) b)^{2}} d t \\
& =b^{-2} \int_{0}^{1}(1-t)^{2}\left(1-t\left(1-\frac{a}{b}\right)\right)^{-2} d t-\int_{0}^{\frac{(x-a) b}{(b-a) x}} \frac{1-2 t+t^{2}}{(t a+(1-t) b)^{2}} d t \\
& =b^{-2} \int_{0}^{1}(1-t)^{2}\left(1-t\left(1-\frac{a}{b}\right)\right)^{-2} d t-\int_{0}^{\frac{(x-a) b}{(b-a) x}} \frac{1}{(t a+(1-t) b)^{2}} d t \\
& +2 \int_{0}^{\frac{(x-a) b}{(b-a) x}} \frac{t}{(t a+(1-t) b)^{2}} d t-\int_{0}^{\frac{(x-a) b}{(b-a) x}} \frac{t^{2}}{(t a+(1-t) b)^{2}} d t \\
& =\left[\begin{array}{c}
\frac{b^{-2}}{3}{ }_{2} F_{1}\left(2,1 ; 4 ;\left(1-\frac{a}{b}\right)\right) \\
-\frac{(x-a) b^{-1}}{(b-a) x}{ }_{2} F_{1}\left(2,1 ; 2 ; \frac{(x-a) b}{(b-a) x}\left(1-\frac{a}{b}\right)\right) \\
+\left(\frac{(x-a)}{(b-a) x}\right)_{2}^{2}{ }_{2} F_{1}\left(2,2 ; 3 ; \frac{(x-a) b}{(b-a) x}\left(1-\frac{a}{b}\right)\right) \\
-\frac{b}{3}\left(\frac{(x-a)}{(b-a) x}\right)^{3}{ }_{2} F_{1}\left(2,3 ; 4 ; \frac{(x-a) b}{(b-a) x}\left(1-\frac{a}{b}\right)\right)
\end{array}\right]=T_{3}(a, b, x), \\
& \int_{\frac{(x-a) b}{(b-a) x}}^{1} \frac{t(1-t)}{(t a+(1-t) b)^{2}} d t=\int_{0}^{1} \frac{t(1-t)}{(t a+(1-t) b)^{2}} d t-\int_{0}^{\frac{(x-a) b}{(b-a) x}} \frac{t(1-t)}{(t a+(1-t) b)^{2}} d t \\
& =b^{-2} \int_{0}^{1} t(1-t)\left(1-t\left(1-\frac{a}{b}\right)\right)^{-2} d t-\int_{0}^{\frac{(x-a) b}{(b-a) x}} \frac{t}{(t a+(1-t) b)^{2}} d t \\
& +\int_{0}^{\frac{(x-a) b}{(b-a) x}} \frac{t^{2}}{(t a+(1-t) b)^{2}} d t \\
& =\left[\begin{array}{c}
\frac{b^{-2}}{6}{ }_{2} F_{1}\left(2,2 ; 4 ;\left(1-\frac{a}{b}\right)\right) \\
-\frac{1}{2}\left(\frac{(x-a)}{(b-a) x}\right)_{2}^{2} F_{1}\left(2,2 ; 3 ; \frac{(x-a) b}{(b-a) x}\left(1-\frac{a}{b}\right)\right) \\
+\frac{b}{3}\left(\frac{(x-a)}{(b-a) x}\right)_{2}^{3}{ }_{2} F_{1}\left(2,3 ; 4 ; \frac{(x-a) b}{(b-a) x}\left(1-\frac{a}{b}\right)\right)
\end{array}\right]=T_{4}(a, b, x) .
\end{aligned}
$$

A combination of (6)-(10) we have (5). This completes the proof. 
Corollary 1. In addition to the conditions of the Theorem 3, if we choose:

1. $\left|f^{\prime}(x)\right| \leq M$, for all $x \in[a, b]$, we have the following Ostrowski's type inequality

$$
\left|f(x)-\frac{a b}{b-a} \int_{a}^{b} \frac{f(u)}{u^{2}} d u\right| \leq a b(b-a) M\left[\begin{array}{c}
T_{1}(a, b, x)+T_{3}(a, b, x) \\
+T_{2}(a, b, x)+T_{4}(a, b, x)
\end{array}\right],
$$

$2 . x=\frac{2 a b}{a+b}$, we have the following midpoint type inequality for harmonically convex functions

$$
\left|f\left(\frac{2 a b}{a+b}\right)-\frac{a b}{b-a} \int_{a}^{b} \frac{f(u)}{u^{2}} d u\right| \leq a b(b-a)\left[\begin{array}{c}
\left|f^{\prime}(a)\right|\left(T_{1}\left(a, b, \frac{2 a b}{a+b}\right)+T_{3}\left(a, b, \frac{2 a b}{a+b}\right)\right) \\
+\left|f^{\prime}(b)\right|\left(T_{2}\left(a, b, \frac{2 a b}{a+b}\right)+T_{4}\left(a, b, \frac{2 a b}{a+b}\right)\right)
\end{array}\right]
$$

Theorem 4. Let $f: I \subset(0, \infty) \rightarrow \mathbb{R}$ be a differentiable function on $I^{\circ}$ such that $a, b \in I$ with $a<b$ and $f^{\prime} \in L$. If $\left|f^{\prime}\right|^{q}$ is harmonically convex on $[a, b]$ for $q \geq 1$, then for all $x \in[a, b]$, we have

$$
\begin{aligned}
\left|f(x)-\frac{a b}{b-a} \int_{a}^{b} \frac{f(u)}{u^{2}} d u\right| \leq & a b(b-a)\left[\left(\frac{1}{2}\left(\frac{(x-a) b}{(b-a) x}\right)^{2}\right)^{1-\frac{1}{q}}\left(\begin{array}{c}
\left|f^{\prime}(a)\right|^{q} T_{5}(a, b, x) \\
+\left|f^{\prime}(b)\right|^{q} T_{6}(a, b, x)
\end{array}\right)^{\frac{1}{q}}\right. \\
& \left.+\left(\frac{1}{2}+\frac{1}{2}\left(\frac{(x-a) b}{(b-a) x}\right)^{2}-\frac{(x-a) b}{(b-a) x}\right)^{1-\frac{1}{q}}\left(\begin{array}{c}
\left|f^{\prime}(a)\right|^{q} T_{7}(a, b, x) \\
+\left|f^{\prime}(b)\right|^{q} T_{8}(a, b, x)
\end{array}\right)^{\frac{1}{q}}\right]
\end{aligned}
$$

where

$$
\begin{aligned}
& T_{5}(a, b, x)=\left[\begin{array}{c}
\frac{1}{2 b^{2 q}}\left(\frac{(x-a) b}{(b-a) x}\right)_{2}^{2}{ }_{2} F_{1}\left(2 q, 2 ; 3 ; \frac{(x-a) b}{(b-a) x}\left(1-\frac{a}{b}\right)\right) \\
-\frac{1}{3 b^{2 q}}\left(\frac{(x-a) b}{(b-a) x}\right)^{3}{ }_{2} F_{1}\left(2 q, 3 ; 4 ; \frac{(x-a) b}{(b-a) x}\left(1-\frac{a}{b}\right)\right)
\end{array}\right] \\
& T_{6}(a, b, x)=\frac{1}{3 b^{2 q}}\left(\frac{(x-a) b}{(b-a) x}\right)_{2}^{3} F_{1}\left(2 q, 3 ; 4 ; \frac{(x-a) b}{(b-a) x}\left(1-\frac{a}{b}\right)\right) \\
& T_{7}(a, b, x)=\left[\begin{array}{c}
\frac{1}{3 b^{2 q}}{ }_{2} F_{1}\left(2 q, 1 ; 4 ;\left(1-\frac{a}{b}\right)\right) \\
-\frac{1}{b^{2 q}} \frac{(x-a) b}{(b-a) x}{ }_{2} F_{1}\left(2 q, 1 ; 2 ; \frac{(x-a) b}{(b-a) x}\left(1-\frac{a}{b}\right)\right) \\
+\frac{1}{b^{2 q}}\left(\frac{(x-a) b}{(b-a) x}\right)_{2}^{2} F_{1}\left(2 q, 2 ; 3 ; \frac{(x-a) b}{(b-a) x}\left(1-\frac{a}{b}\right)\right) \\
-\frac{1}{3 b^{2 q}}\left(\frac{(x-a) b}{(b-a) x}\right)^{3}{ }_{2} F_{1}\left(2 q, 3 ; 4 ; \frac{(x-a) b}{(b-a) x}\left(1-\frac{a}{b}\right)\right)
\end{array}\right] \\
& T_{8}(a, b, x)=\left[\begin{array}{c}
\frac{1}{6 b^{2 q}}{ }_{2} F_{1}\left(2 q, 2 ; 4 ;\left(1-\frac{a}{b}\right)\right) \\
-\frac{1}{2 b^{2 q}}\left(\frac{(x-a) b}{(b-a) x}\right)_{2}{ }_{2} F_{1}\left(2 q, 2 ; 3 ; \frac{(x-a) b}{(b-a) x}\left(1-\frac{a}{b}\right)\right) \\
+\frac{1}{3 b^{2 q}}\left(\frac{(x-a) b}{(b-a) x}\right)_{2}^{3}{ }_{2} F_{1}\left(2,3 ; 4 ; \frac{(x-a) b}{(b-a) x}\left(1-\frac{a}{b}\right)\right)
\end{array}\right] .
\end{aligned}
$$

Proof. By using Lemma 1, power mean inequality and harmonically convexity of $\left|f^{\prime}\right|^{q}$, we have

$$
\left|f(x)-\frac{a b}{b-a} \int_{a}^{b} \frac{f(u)}{u^{2}} d u\right| \leq a b(b-a)\left[\int_{0}^{\frac{(x-a) b}{(b-a) x}} \frac{t}{(t a+(1-t) b)^{2}}\left|f^{\prime}\left(\frac{a b}{t a+(1-t) b}\right)\right| d t\right.
$$




$$
\begin{aligned}
& \left.+\int_{\frac{(x-a) b}{(b-a) x}}^{1} \frac{1-t}{(t a+(1-t) b)^{2}}\left|f^{\prime}\left(\frac{a b}{t a+(1-t) b}\right)\right| d t\right] \\
& \leq a b(b-a)\left[\left(\int_{0}^{\frac{(x-a) b}{(b-a) x}} t d t\right)^{1-\frac{1}{q}}\left(\int_{0}^{\frac{(x-a) b}{(b-a) x}} \frac{t}{(t a+(1-t) b)^{2 q}}\left[(1-t)\left|f^{\prime}(a)\right|^{q}+t\left|f^{\prime}(b)\right|^{q}\right] d t\right)^{\frac{1}{q}}\right. \\
& \left.+\left(\int_{\frac{(x-a) b}{(b-a) x}}^{1}(1-t) d t\right)^{1-\frac{1}{q}}\left(\int_{\frac{(x-a) b}{(b-a) x}}^{1} \frac{1-t}{(t a+(1-t) b)^{2 q}}\left[(1-t)\left|f^{\prime}(a)\right|^{q}+t\left|f^{\prime}(b)\right|^{q}\right] d t\right)^{\frac{1}{q}}\right] \\
& \leq a b(b-a)\left[\left(\frac{1}{2}\left(\frac{(x-a) b}{(b-a) x}\right)^{2}\right)^{1-\frac{1}{q}}\left(\begin{array}{c}
\left|f^{\prime}(a)\right|^{q} \int_{0}^{\frac{(x-a) b}{(b-a) x}} \frac{t(1-t)}{(t a+(1-t) b)^{2 q}} d t \\
+\left|f^{\prime}(b)\right|^{q} \int_{0}^{\frac{(x-a) b}{(b-a) x}} \frac{t^{2}}{(t a+(1-t) b)^{2 q}} d t
\end{array}\right)^{\frac{1}{q}}\right. \\
& \left.+\left(\frac{1}{2}+\frac{1}{2}\left(\frac{(x-a) b}{(b-a) x}\right)^{2}-\frac{(x-a) b}{(b-a) x}\right)^{1-\frac{1}{q}}\left(\begin{array}{c}
\left|f^{\prime}(a)\right|^{q} \int_{\frac{(x-a) b}{(b-a) x}}^{1} \frac{(1-t)^{2}}{(t a+(1-t) b)^{2 q}} d t \\
+\left|f^{\prime}(b)\right|^{q} \int_{\frac{(x-a) b}{(b-a) x}}^{1} \frac{t(1-t)}{(t a+(1-t) b)^{2 q}} d t
\end{array}\right)^{\frac{1}{q}}\right]
\end{aligned}
$$

Calculating appearing integrals with hypergeometric functions, we have

$$
\begin{aligned}
& \int_{0}^{\frac{(x-a) b}{(b-a) x}} \frac{t(1-t)}{(t a+(1-t) b)^{2 q}} d t=\left[\begin{array}{c}
\frac{1}{2 b^{2 q}}\left(\frac{(x-a) b}{(b-a) x}\right)_{2}^{2}{ }_{2} F_{1}\left(2 q, 2 ; 3 ; \frac{(x-a) b}{(b-a) x}\left(1-\frac{a}{b}\right)\right) \\
-\frac{1}{3 b^{2 q}}\left(\frac{(x-a) b}{(b-a) x}\right)^{3}{ }_{2} F_{1}\left(2 q, 3 ; 4 ; \frac{(x-a) b}{(b-a) x}\left(1-\frac{a}{b}\right)\right)
\end{array}\right]=T_{5}(a, b, x), \\
& \int_{0}^{\frac{(x-a) b}{(b-a) x}} \frac{t^{2}}{(t a+(1-t) b)^{2 q}} d t=\frac{1}{3 b^{2 q}}\left(\frac{(x-a) b}{(b-a) x}\right)^{3}{ }_{2} F_{1}\left(2 q, 3 ; 4 ; \frac{(x-a) b}{(b-a) x}\left(1-\frac{a}{b}\right)\right)=T_{6}(a, b, x), \\
& \int_{\frac{(x-a) b}{(b-a) x}}^{1} \frac{(1-t)^{2}}{(t a+(1-t) b)^{2 q}} d t=\left[\begin{array}{c}
\frac{1}{3 b^{2 q}}{ }_{2} F_{1}\left(2 q, 1 ; 4 ;\left(1-\frac{a}{b}\right)\right) \\
-\frac{1}{b^{2 q}} \frac{(x-a) b}{(b-a) x}{ }_{2} F_{1}\left(2 q, 1 ; 2 ; \frac{(x-a) b}{(b-a) x}\left(1-\frac{a}{b}\right)\right) \\
+\frac{1}{b^{2 q}}\left(\frac{(x-a) b}{(b-a) x}\right)_{2}^{2}{ }_{2} F_{1}\left(2 q, 2 ; 3 ; \frac{(x-a) b}{(b-a) x}\left(1-\frac{a}{b}\right)\right) \\
-\frac{1}{3 b^{2 q}}\left(\frac{(x-a) b}{(b-a) x}\right)_{2}^{3}{ }_{2} F_{1}\left(2 q, 3 ; 4 ; \frac{(x-a) b}{(b-a) x}\left(1-\frac{a}{b}\right)\right)
\end{array}\right]=T_{7}(a, b, x) \\
& \int_{\frac{(x-a) b}{(b-a) x}}^{1} \frac{t(1-t)}{(t a+(1-t) b)^{2 q}} d t=\left[\begin{array}{c}
\frac{1}{6 b^{2 q}}{ }_{2} F_{1}\left(2 q, 2 ; 4 ;\left(1-\frac{a}{b}\right)\right) \\
-\frac{1}{2 b^{2 q}}\left(\frac{(x-a) b}{(b-a) x}\right)_{2}^{2} F_{1}\left(2 q, 2 ; 3 ; \frac{(x-a) b}{(b-a) x}\left(1-\frac{a}{b}\right)\right) \\
+\frac{1}{3 b^{2 q}}\left(\frac{(x-a) b}{(b-a) x}\right)^{3}{ }_{2} F_{1}\left(2,3 ; 4 ; \frac{(x-a) b}{(b-a) x}\left(1-\frac{a}{b}\right)\right)
\end{array}\right]=T_{8}(a, b, x) .
\end{aligned}
$$

A combination of (14)-(18) we have (13). This completes the proof.

Corollary 2. In addition to the conditions of the Theorem 4, if we choose: 
(1) $\left|f^{\prime}(x)\right| \leq M$, for all $x \in[a, b]$, we have the following Ostrowski's type inequality

$$
\begin{aligned}
\left|f(x)-\frac{a b}{b-a} \int_{a}^{b} \frac{f(u)}{u^{2}} d u\right| & \leq a b(b-a) M\left[\left(\frac{1}{2}\left(\frac{(x-a) b}{(b-a) x}\right)^{2}\right)^{1-\frac{1}{q}}\left(T_{5}(a, b, x)+T_{6}(a, b, x)\right)^{\frac{1}{q}}\right. \\
& \left.+\left(\frac{1}{2}+\frac{1}{2}\left(\frac{(x-a) b}{(b-a) x}\right)^{2}-\frac{(x-a) b}{(b-a) x}\right)^{1-\frac{1}{q}}\left(T_{7}(a, b, x)+T_{8}(a, b, x)\right)^{\frac{1}{q}}\right]
\end{aligned}
$$

(2) $x=\frac{2 a b}{a+b}$, we have the following midpoint type inequality for harmonically convex functions

$$
\begin{aligned}
\left|f\left(\frac{2 a b}{a+b}\right)-\frac{a b}{b-a} \int_{a}^{b} \frac{f(u)}{u^{2}} d u\right| & \leq a b(b-a)\left(\frac{1}{8}\right)^{1-\frac{1}{q}}\left[\left(\left|f^{\prime}(a)\right|^{q} T_{5}\left(a, b, \frac{2 a b}{a+b}\right)+\left|f^{\prime}(b)\right|^{q} T_{6}\left(a, b, \frac{2 a b}{a+b}\right)\right)^{\frac{1}{q}}\right. \\
& \left.+\left(\left|f^{\prime}(a)\right|^{q} T_{7}\left(a, b, \frac{2 a b}{a+b}\right)+\left|f^{\prime}(b)\right|^{q} T_{8}\left(a, b, \frac{2 a b}{a+b}\right)\right)^{\frac{1}{q}}\right] .
\end{aligned}
$$

Theorem 5. Let $f: I \subset(0, \infty) \rightarrow \mathbb{R}$ be a differentiable function on $I^{\circ}$ such that $a, b \in I$ with $a<b$ and $f^{\prime} \in L$. If $\left|f^{\prime}\right|^{q}$ is harmonically convex on $[a, b]$ for $q \geq 1$, then for all $x \in[a, b]$, we have

$$
\begin{aligned}
\left|f(x)-\frac{a b}{b-a} \int_{a}^{b} \frac{f(u)}{u^{2}} d u\right| & \leq a b(b-a)\left[\left(T_{9}(a, b, x)\right)^{1-\frac{1}{q}}\left(\left|f^{\prime}(a)\right|^{q} T_{1}(a, b, x)+\left|f^{\prime}(b)\right|^{q} T_{2}(a, b, x)\right)^{\frac{1}{q}}\right. \\
& \left.+\left(T_{10}(a, b, x)\right)^{1-\frac{1}{q}}\left(\left|f^{\prime}(a)\right|^{q} T_{3}(a, b, x)+\left|f^{\prime}(b)\right|^{q} T_{4}(a, b, x)\right)^{\frac{1}{q}}\right]
\end{aligned}
$$

where $T_{1}(a, b, x)-T_{4}(a, b, x)$ are defined as in Theorem 3 and

$$
\begin{gathered}
T_{9}(a, b, x)=\frac{1}{2}\left(\frac{(x-a)}{(b-a) x}\right)^{2}{ }_{2} F_{1}\left(2,2 ; 3 ; \frac{(x-a) b}{(b-a) x}\left(1-\frac{a}{b}\right)\right), \\
T_{10}(a, b, x)=\left[\begin{array}{c}
-\frac{(x-a) b^{-1}}{(b-a) x}{ }_{2} F_{1}\left(2,1 ; 2 ; \frac{(x-a) b}{(b-a) x}\left(1-\frac{a}{b}\right)\right) \\
+\frac{1}{2}\left(\frac{(x-a)}{(b-a) x}\right)^{2}{ }_{2} F_{1}\left(2,2 ; 3 ; \frac{(x-a) b}{(b-a) x}\left(1-\frac{a}{b}\right)\right)
\end{array}\right] .
\end{gathered}
$$

Proof. By using Lemma 1, power mean inequality and harmonically convexity of $\left|f^{\prime}\right|^{q}$, we have

$$
\begin{aligned}
& \left|f(x)-\frac{a b}{b-a} \int_{a}^{b} \frac{f(u)}{u^{2}} d u\right| \leq a b(b-a)\left[\int_{0}^{\frac{(x-a) b}{(b-a) x}} \frac{t}{(t a+(1-t) b)^{2}}\left|f^{\prime}\left(\frac{a b}{t a+(1-t) b}\right)\right| d t\right. \\
& \left.+\int_{\frac{(x-a) b}{(b-a) x}}^{1} \frac{1-t}{(t a+(1-t) b)^{2}}\left|f^{\prime}\left(\frac{a b}{t a+(1-t) b}\right)\right| d t\right] \\
& \leq a b(b-a)\left[\left(\int_{0}^{\frac{(x-a) b}{(b-a) x}} \frac{t}{(t a+(1-t) b)^{2}} d t\right)^{1-\frac{1}{q}}\left(\int_{0}^{\frac{(x-a) b}{(b-a) x}} \frac{t}{(t a+(1-t) b)^{2}}\left[(1-t)\left|f^{\prime}(a)\right|^{q}+t\left|f^{\prime}(b)\right|^{q}\right] d t\right)^{\frac{1}{q}}\right. \\
& \left.+\left(\int_{\frac{(x-a) b}{(b-a) x}}^{1} \frac{1-t}{(t a+(1-t) b)^{2}} d t\right)^{1-\frac{1}{q}}\left(\int_{\frac{(x-a) b}{(b-a) x}}^{1} \frac{1-t}{(t a+(1-t) b)^{2}}\left[(1-t)\left|f^{\prime}(a)\right|^{q}+t\left|f^{\prime}(b)\right|^{q}\right] d t\right)^{\frac{1}{q}}\right]
\end{aligned}
$$




$$
\begin{aligned}
& \leq a b(b-a)\left[\left(\int_{0}^{\frac{(x-a) b}{(b-a) x}} \frac{t}{(t a+(1-t) b)^{2}} d t\right)^{1-\frac{1}{q}}\left(\begin{array}{c}
\left|f^{\prime}(a)\right|^{q} \int_{0}^{\frac{(x-a) b}{(b-a) x}} \frac{t(1-t)}{(t a+(1-t) b)^{2 q}} d t \\
+\left|f^{\prime}(b)\right|^{q} \int_{0}^{\frac{(x-a) b}{(b-a) x}} \frac{t^{2}}{(t a+(1-t) b)^{2 q}} d t
\end{array}\right)^{\frac{1}{q}}\right. \\
& \left.+\left(\int_{\frac{(x-a) b}{(b-a) x}}^{1} \frac{1-t}{(t a+(1-t) b)^{2}} d t\right)^{1-\frac{1}{q}}\left(\begin{array}{c}
\left|f^{\prime}(a)\right|^{q} \int_{\frac{(x-a) b}{(b-a) x}}^{1} \frac{(1-t)^{2}}{(t a+(1-t) b)^{2 q}} d t \\
+\left|f^{\prime}(b)\right|^{q} \int_{\frac{(x-a) b}{(b-a) x}}^{1} \frac{t(1-t)}{(t a+(1-t) b)^{2 q}} d t
\end{array}\right)^{\frac{1}{q}}\right] .
\end{aligned}
$$

Calculating appearing integrals with hypergeometric functions, we have

$$
\begin{gathered}
\int_{0}^{\frac{(x-a) b}{(b-a) x}} \frac{t}{(t a+(1-t) b)^{2}} d t=\frac{1}{2}\left(\frac{(x-a)}{(b-a) x}\right)^{2}{ }_{2} F_{1}\left(2,2 ; 3 ; \frac{(x-a) b}{(b-a) x}\left(1-\frac{a}{b}\right)\right)=T_{9}(a, b, x), \\
\int_{\frac{(x-a) b}{(b-a) x}}^{1} \frac{1-t}{(t a+(1-t) b)^{2}} d t=\left[\begin{array}{c}
\frac{b^{-2}}{2}{ }_{2} F_{1}\left(2,1 ; 3 ;\left(1-\frac{a}{b}\right)\right) \\
-\frac{(x-a) b^{-1}}{(b-a) x}{ }_{2} F_{1}\left(2,1 ; 2 ; \frac{(x-a) b}{(b-a) x}\left(1-\frac{a}{b}\right)\right) \\
+\frac{1}{2}\left(\frac{(x-a)}{(b-a) x}\right)_{2}{ }_{2} F_{1}\left(2,2 ; 3 ; \frac{(x-a) b}{(b-a) x}\left(1-\frac{a}{b}\right)\right)
\end{array}\right]=T_{10}(a, b, x) .
\end{gathered}
$$

A combination of (7)-(10) and (22)-(24)we have (21). This completes the proof.

Corollary 3. In addition to the conditions of the Theorem 5, if we choose:

(1) $\left|f^{\prime}(x)\right| \leq M$, for all $x \in[a, b]$, we have the following Ostrowski's type inequality

$$
\begin{aligned}
\left|f(x)-\frac{a b}{b-a} \int_{a}^{b} \frac{f(u)}{u^{2}} d u\right| & \leq a b(b-a) M\left[\left(T_{9}(a, b, x)\right)^{1-\frac{1}{q}}\left(T_{1}(a, b, x)+T_{2}(a, b, x)\right)^{\frac{1}{q}}\right. \\
& \left.+\left(T_{10}(a, b, x)\right)^{1-\frac{1}{q}}\left(T_{3}(a, b, x)+T_{4}(a, b, x)\right)^{\frac{1}{q}}\right]
\end{aligned}
$$

(2) $x=\frac{2 a b}{a+b}$, we have the following midpoint type inequality for harmonically convex functions

$$
\begin{aligned}
& \left|f\left(\frac{2 a b}{a+b}\right)-\frac{a b}{b-a} \int_{a}^{b} \frac{f(u)}{u^{2}} d u\right| \\
& \leq a b(b-a)\left[\left(T_{9}\left(a, b, \frac{2 a b}{a+b}\right)\right)^{1-\frac{1}{q}}\left(\left|f^{\prime}(a)\right|^{q} T_{1}\left(a, b, \frac{2 a b}{a+b}\right)+\left|f^{\prime}(b)\right|^{q} T_{2}\left(a, b, \frac{2 a b}{a+b}\right)\right)^{\frac{1}{q}}\right. \\
& \left.+\left(T_{10}\left(a, b, \frac{2 a b}{a+b}\right)\right)^{1-\frac{1}{q}}\left(\left|f^{\prime}(a)\right|^{q} T_{3}\left(a, b, \frac{2 a b}{a+b}\right)+\left|f^{\prime}(b)\right|^{q} T_{4}\left(a, b, \frac{2 a b}{a+b}\right)\right)^{\frac{1}{q}}\right] .
\end{aligned}
$$

Theorem 6. Let $f: I \subset(0, \infty) \rightarrow \mathbb{R}$ be a differentiable function on $I^{\circ}$ such that $a, b \in I$ with $a<b$ and $f^{\prime} \in L$. If $\left|f^{\prime}\right|^{q}$ is harmonically convex on $[a, b]$ for $q>1$ and $\frac{1}{p}+\frac{1}{q}=1$, then for all $x \in[a, b]$, we have

$$
\begin{aligned}
\left|f(x)-\frac{a b}{b-a} \int_{a}^{b} \frac{f(u)}{u^{2}} d u\right| & \leq a b(b-a)\left[\left(\frac{1}{p+1}\left(\frac{(x-a) b}{(b-a) x}\right)^{p+1}\right)^{\frac{1}{p}}\left(\begin{array}{c}
\left|f^{\prime}(a)\right|^{q} T_{11}(a, b, x) \\
+\left|f^{\prime}(b)\right|^{q} T_{12}(a, b, x)
\end{array}\right)^{\frac{1}{q}}\right. \\
& \left.+\left(\frac{1}{p+1}\left(\frac{(b-x) a}{(b-a) x}\right)^{p+1}\right)^{\frac{1}{p}}\left(\begin{array}{c}
\left|f^{\prime}(a)\right|^{q} T_{13}(a, b, x) \\
+\left|f^{\prime}(b)\right|^{q} T_{14}(a, b, x)
\end{array}\right)^{\frac{1}{q}}\right]
\end{aligned}
$$


where

$$
\begin{aligned}
& T_{11}(a, b, x)=\left[\begin{array}{c}
\frac{1}{2 b^{2 q}}\left(\frac{(x-a) b}{(b-a) x}\right){ }_{2} F_{1}\left(2 q, 1 ; 2 ; \frac{(x-a) b}{(b-a) x}\left(1-\frac{a}{b}\right)\right) \\
-\frac{1}{2 b^{2 q}}\left(\frac{(x-a) b}{(b-a) x}\right)_{2}^{2}{ }_{2} F_{1}\left(2 q, 2 ; 3 ; \frac{(x-a) b}{(b-a) x}\left(1-\frac{a}{b}\right)\right)
\end{array}\right], \\
& T_{12}(a, b, x)=\frac{1}{2 b^{2 q}}\left(\frac{(x-a) b}{(b-a) x}\right)^{2}{ }_{2} F_{1}\left(2 q, 2 ; 3 ; \frac{(x-a) b}{(b-a) x}\left(1-\frac{a}{b}\right)\right), \\
& T_{13}(a, b, x)=\left[\begin{array}{c}
-\frac{1}{2 b^{2 q}}\left(\frac{(x-a) b}{(b-a) x}\right){ }_{2} F_{1}\left(2 q, 1 ; 2 ; \frac{(x-a) b}{(b-a) x}\left(1-\frac{a}{b}\right)\right) \\
+\frac{1}{2 b^{2 q}}\left(\frac{(x-a) b}{(b-a) x}\right)_{2}^{2}{ }_{2} F_{1}\left(2 q, 2 ; 3 ; \frac{(x-a) b}{(b-a) x}\left(1-\frac{a}{b}\right)\right)
\end{array}\right], \\
& T_{14}(a, b, x)=\left[\begin{array}{c}
\frac{1}{2 b^{2 q}} 2 F_{1}\left(2 q, 2 ; 3 ;\left(1-\frac{a}{b}\right)\right) \\
-\frac{1}{2 b^{2 q}}\left(\frac{(x-a) b}{(b-a) x}\right)^{2}{ }_{2} F_{1}\left(2 q, 2 ; 3 ; \frac{(x-a) b}{(b-a) x}\left(1-\frac{a}{b}\right)\right)
\end{array}\right] .
\end{aligned}
$$

Proof. By using Lemma 1, Hölder inequality and harmonically convexity of $\left|f^{\prime}\right|^{q}$, we have

$$
\begin{aligned}
& \left|f(x)-\frac{a b}{b-a} \int_{a}^{b} \frac{f(u)}{u^{2}} d u\right| \\
& \leq a b(b-a)\left[\int_{0}^{\frac{(x-a) b}{(b-a) x}} \frac{t}{(t a+(1-t) b)^{2}}\left|f^{\prime}\left(\frac{a b}{t a+(1-t) b}\right)\right| d t+\int_{\frac{(x-a) b}{(b-a) x}}^{1} \frac{1-t}{(t a+(1-t) b)^{2}}\left|f^{\prime}\left(\frac{a b}{t a+(1-t) b}\right)\right| d t\right] \\
& \leq a b(b-a)\left[\left(\int_{0}^{\frac{(x-a) b}{(b-a) x}} t^{p} d t\right)^{\frac{1}{p}}\left(\int_{0}^{\frac{(x-a) b}{(b-a) x}} \frac{1}{(t a+(1-t) b)^{2 q}}\left[(1-t)\left|f^{\prime}(a)\right|^{q}+t\left|f^{\prime}(b)\right|^{q}\right] d t\right)^{\frac{1}{q}}\right. \\
& \left.+\left(\int_{\frac{(x-a) b}{(b-a) x}}^{1}(1-t)^{p} d t\right)^{\frac{1}{p}}\left(\int_{\frac{(x-a) b}{(b-a) x}}^{1} \frac{1-t}{(t a+(1-t) b)^{2 q}}\left[(1-t)\left|f^{\prime}(a)\right|^{q}+t\left|f^{\prime}(b)\right|^{q}\right] d t\right)^{\frac{1}{q}}\right] \\
& \leq a b(b-a)\left[\left(\frac{1}{p+1}\left(\frac{(x-a) b}{(b-a) x}\right)^{p+1}\right)^{\frac{1}{p}}\left(\begin{array}{c}
\left|f^{\prime}(a)\right|^{q} \int_{0}^{\frac{(x-a) b}{(b-a) x}} \frac{1-t}{(t a+(1-t) b)^{2 q}} d t \\
+\left|f^{\prime}(b)\right|^{q} \int_{0}^{\frac{(x-a) b}{(b-a)}} \frac{t}{(t a+(1-t) b)^{2 q}} d t
\end{array}\right)^{\frac{1}{q}}\right. \\
& \left.+\left(\frac{1}{p+1}\left(\frac{(b-x) a}{(b-a) x}\right)^{p+1}\right)^{\frac{1}{p}}\left(\begin{array}{c}
\left|f^{\prime}(a)\right|^{q} \int_{\frac{(x-a) b}{(b-a x)}}^{1} \frac{1-t}{(t a+(1-t) b)^{2 q}} d t \\
+\left|f^{\prime}(b)\right|^{q} \int_{\frac{(x-a) b}{(b-a) x}}^{1} \frac{t}{(t a+(1-t) b)^{2 q}} d t
\end{array}\right)^{\frac{1}{q}}\right] .
\end{aligned}
$$

Calculating appearing integrals with hypergeometric functions, we have

$$
\int_{0}^{\frac{(x-a) b}{(b-a) x}} \frac{1-t}{(t a+(1-t) b)^{2 q}} d t=\left[\begin{array}{c}
\frac{1}{2 b^{2 q}}\left(\frac{(x-a) b}{(b-a) x}\right){ }_{2} F_{1}\left(2 q, 1 ; 2 ; \frac{(x-a) b}{(b-a) x}\left(1-\frac{a}{b}\right)\right) \\
-\frac{1}{2 b^{2 q}}\left(\frac{(x-a) b}{(b-a) x}\right)_{2}^{2}{ }_{2} F_{1}\left(2 q, 2 ; 3 ; \frac{(x-a) b}{(b-a) x}\left(1-\frac{a}{b}\right)\right)
\end{array}\right]=T_{11}(a, b, x),
$$




$$
\begin{aligned}
& \int_{0}^{\frac{(x-a) b}{(b-a) x}} \frac{t}{(t a+(1-t) b)^{2 q}} d t=\frac{1}{2 b^{2 q}}\left(\frac{(x-a) b}{(b-a) x}\right)^{2}{ }_{2} F_{1}\left(2 q, 2 ; 3 ; \frac{(x-a) b}{(b-a) x}\left(1-\frac{a}{b}\right)\right)=T_{12}(a, b, x), \\
& \int_{\frac{(x-a) b}{(b-a) x} \frac{1-t}{(t a+(1-t) b)^{2 q}} d t=}^{1}\left[\begin{array}{l}
-\frac{1}{2 b^{2 q}}\left(\frac{(x-a) b}{(b-a) x}\right){ }_{2} F_{1}\left(2 q, 1 ; 2 ; \frac{(x-a) b}{(b-a) x}\left(1-\frac{a}{b}\right)\right) \\
+\frac{1}{2 b^{2 q}}\left(\frac{(x-a) b}{(b-a) x}\right)_{2}{ }_{2} F_{1}\left(2 q, 2 ; 3 ; \frac{(x-a) b}{(b-a) x}\left(1-\frac{a}{b}\right)\right)
\end{array}\right]=T_{13}(a, b, x), \\
& \int_{\frac{(x-a) b}{(b-a) x}}^{1} \frac{t}{(t a+(1-t) b)^{2 q}} d t=\left[\begin{array}{c}
\frac{1}{2 b^{2 q}}{ }_{2} F_{1}\left(2 q, 2 ; 3 ;\left(1-\frac{a}{b}\right)\right) \\
-\frac{1}{2 b^{2 q}}\left(\frac{(x-a) b}{(b-a) x}\right)^{2}{ }_{2} F_{1}\left(2 q, 2 ; 3 ; \frac{(x-a) b}{(b-a) x}\left(1-\frac{a}{b}\right)\right)
\end{array}\right]=T_{14}(a, b, x) .
\end{aligned}
$$

A combination of (28)-(32) we have (27). This completes the proof.

Corollary 4. In addition to the conditions of the Theorem 6, if we choose:

(1) $\left|f^{\prime}(x)\right| \leq M$, for all $x \in[a, b]$, we have the following Ostrowski's type inequality

$$
\begin{aligned}
\left|f(x)-\frac{a b}{b-a} \int_{a}^{b} \frac{f(u)}{u^{2}} d u\right| & \leq a b(b-a) M\left[\left(\frac{1}{p+1}\left(\frac{(x-a) b}{(b-a) x}\right)^{p+1}\right)^{\frac{1}{p}}\left(T_{11}(a, b, x)+T_{12}(a, b, x)\right)^{\frac{1}{q}}\right. \\
& \left.+\left(\frac{1}{p+1}\left(\frac{(b-x) a}{(b-a) x}\right)^{p+1}\right)^{\frac{1}{p}}\left(T_{13}(a, b, x)+T_{14}(a, b, x)\right)^{\frac{1}{q}}\right],
\end{aligned}
$$

(2) $x=\frac{2 a b}{a+b}$, we have the following midpoint type inequality for harmonically convex functions

$$
\begin{aligned}
\left|f\left(\frac{2 a b}{a+b}\right)-\frac{a b}{b-a} \int_{a}^{b} \frac{f(u)}{u^{2}} d u\right| & \leq a b(b-a)\left[\left(\begin{array}{c}
1 \\
p+1
\end{array}\right)^{\frac{1}{p}}\left(\begin{array}{c}
\left|f^{\prime}(a)\right|^{q} T_{11}\left(a, b, \frac{2 a b}{a+b}\right) \\
+\left|f^{\prime}(b)\right|^{q} T_{12}\left(a, b, \frac{2 a b}{a+b}\right)
\end{array}\right)^{\frac{1}{q}}\right. \\
& \left.+\left(\frac{1}{p+1}\right)^{\frac{1}{p}}\left(\begin{array}{c}
\left|f^{\prime}(a)\right|^{q} T_{13}\left(a, b, \frac{2 a b}{a+b}\right) \\
+\left|f^{\prime}(b)\right|^{q} T_{14}\left(a, b, \frac{2 a b}{a+b}\right)
\end{array}\right)^{\frac{1}{q}}\right] .
\end{aligned}
$$

Theorem 7. Let $f: I \subset(0, \infty) \rightarrow \mathbb{R}$ be a differentiable function on $I^{\circ}$ such that $a, b \in I$ with $a<b$ and $f^{\prime} \in L$. If $\left|f^{\prime}\right|^{q}$ is harmonically convex on $[a, b]$ for $q>1$ and $\frac{1}{p}+\frac{1}{q}=1$, then for all $x \in[a, b]$, we have

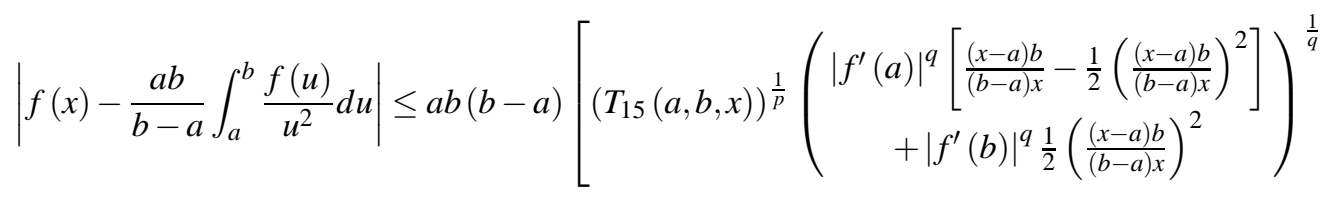

$$
\begin{aligned}
& \left.+\left(T_{16}(a, b, x)\right)^{\frac{1}{p}}\left(\begin{array}{c}
\left|f^{\prime}(a)\right|^{q}\left[\frac{1}{2}-\frac{(x-a) b}{(b-a) x}+\frac{1}{2}\left(\frac{(x-a) b}{(b-a) x}\right)^{2}\right] \\
+\left|f^{\prime}(b)\right|^{q}\left[\frac{1}{2}-\frac{1}{2}\left(\frac{(x-a) b}{(b-a) x}\right)^{2}\right]
\end{array}\right)^{\frac{1}{q}}\right]
\end{aligned}
$$


where

$$
\begin{aligned}
& T_{15}(a, b, x)=\frac{1}{2 b^{2 p}}\left(\frac{(x-a) b}{(b-a) x}\right)^{p+1}{ }_{2} F_{1}\left(2 p, 2 ; 3 ; \frac{(x-a) b}{(b-a) x}\left(1-\frac{a}{b}\right)\right), \\
& T_{16}(a, b, x)=\int_{\frac{(x-a) b}{(b-a) x}}^{1} \frac{(1-t)^{p}}{(t a+(1-t) b)^{2 p}} d t .
\end{aligned}
$$

Proof. By using Lemma 1, Hölder inequality and harmonically convexity of $\left|f^{\prime}\right|^{q}$, we have

$$
\begin{aligned}
& \left|f(x)-\frac{a b}{b-a} \int_{a}^{b} \frac{f(u)}{u^{2}} d u\right| \leq a b(b-a)\left[\int_{0}^{\frac{(x-a) b}{(b-a) x}} \frac{t}{(t a+(1-t) b)^{2}}\left|f^{\prime}\left(\frac{a b}{t a+(1-t) b}\right)\right| d t\right. \\
& \left.+\int_{\frac{(x-a) b}{(b-a) x}}^{1} \frac{1-t}{(t a+(1-t) b)^{2}}\left|f^{\prime}\left(\frac{a b}{t a+(1-t) b}\right)\right| d t\right] \\
& \leq a b(b-a)\left[\left(\int_{0}^{\frac{(x-a) b}{(b-a) x}} \frac{t^{p}}{(t a+(1-t) b)^{2 p}} d t\right)^{\frac{1}{p}}\left(\int_{0}^{\frac{(x-a) b}{(b-a) x}}\left[(1-t)\left|f^{\prime}(a)\right|^{q}+t\left|f^{\prime}(b)\right|^{q}\right] d t\right)^{\frac{1}{q}}\right. \\
& \left.+\left(\int_{\frac{(x-a) b}{(b-a) x}}^{1} \frac{(1-t)^{p}}{(t a+(1-t) b)^{2 p}} d t\right)^{\frac{1}{p}}\left(\int_{\frac{(x-a) b}{(b-a) x}}^{1}\left[(1-t)\left|f^{\prime}(a)\right|^{q}+t\left|f^{\prime}(b)\right|^{q}\right] d t\right)^{\frac{1}{q}}\right] \\
& \leq a b(b-a)\left[\left(\int_{0}^{\frac{(x-a) b}{(b-a) x}} \frac{t^{p}}{(t a+(1-t) b)^{2 p}} d t\right)^{\frac{1}{p}}\left(\begin{array}{c}
\left|f^{\prime}(a)\right|^{q}\left[\frac{(x-a) b}{(b-a) x}-\frac{1}{2}\left(\frac{(x-a) b}{(b-a) x}\right)^{2}\right. \\
+\left|f^{\prime}(b)\right|^{q} \frac{1}{2}\left(\frac{(x-a) b}{(b-a) x}\right)^{2}
\end{array}\right)^{\frac{1}{q}}\right. \\
& \left.+\left(\int_{\frac{(x-a) b}{(b-a) x}}^{1} \frac{(1-t)^{p}}{(t a+(1-t) b)^{2 p}} d t\right)^{\frac{1}{p}}\left(\begin{array}{c}
\left|f^{\prime}(a)\right|^{q}\left[\frac{1}{2}-\frac{(x-a) b}{(b-a) x}+\frac{1}{2}\left(\frac{(x-a) b}{(b-a) x}\right)^{2}\right] \\
+\left|f^{\prime}(b)\right|^{q}\left[\frac{1}{2}-\frac{1}{2}\left(\frac{(x-a) b}{(b-a) x}\right)^{2}\right]
\end{array}\right)^{\frac{1}{q}}\right] .
\end{aligned}
$$

Since the appearing integrals are as the following, we have

$$
\begin{gathered}
\int_{0}^{\frac{(x-a) b}{(b-a) x}} \frac{t^{p}}{(t a+(1-t) b)^{2 p}} d t=\frac{1}{2 b^{2 p}}\left(\frac{(x-a) b}{(b-a) x}\right)^{p+1}{ }_{2} F_{1}\left(2 p, 2 ; 3 ; \frac{(x-a) b}{(b-a) x}\left(1-\frac{a}{b}\right)\right)=T_{15}(a, b, x), \\
\int_{\frac{(x-a) b}{(b-a) x}}^{1} \frac{(1-t)^{p}}{(t a+(1-t) b)^{2 p}} d t=T_{16}(a, b, x) .
\end{gathered}
$$

A combination of (36)-(38) we have (35). This completes the proof.

Corollary 5. In addition to the conditions of the Theorem 5, if we choose:

(1) $\left|f^{\prime}(x)\right| \leq M$, for all $x \in[a, b]$, we have the following Ostrowski's type inequality

$$
\begin{aligned}
\left|f(x)-\frac{a b}{b-a} \int_{a}^{b} \frac{f(u)}{u^{2}} d u\right| & \leq a b(b-a) M\left[\left(T_{15}(a, b, x)\right)^{\frac{1}{p}}\left(\left[\frac{(x-a) b}{(b-a) x}-\frac{1}{2}\left(\frac{(x-a) b}{(b-a) x}\right)^{2}\right]+\frac{1}{2}\left(\frac{(x-a) b}{(b-a) x}\right)^{2}\right)^{\frac{1}{q}}\right. \\
& \left.+\left(T_{16}(a, b, x)\right)^{\frac{1}{p}}\left(\left[\frac{1}{2}-\frac{(x-a) b}{(b-a) x}+\frac{1}{2}\left(\frac{(x-a) b}{(b-a) x}\right)^{2}\right]+\left[\frac{1}{2}-\frac{1}{2}\left(\frac{(x-a) b}{(b-a) x}\right)^{2}\right]\right)^{\frac{1}{q}}\right],
\end{aligned}
$$


(2) $x=\frac{2 a b}{a+b}$, we have the following midpoint type inequality for harmonically convex functions

$$
\begin{aligned}
\left|f\left(\frac{2 a b}{a+b}\right)-\frac{a b}{b-a} \int_{a}^{b} \frac{f(u)}{u^{2}} d u\right| & \leq a b(b-a)\left[\left(T_{15}\left(a, b, \frac{2 a b}{a+b}\right)\right)^{\frac{1}{p}}\left(\frac{3\left|f^{\prime}(a)\right|^{q}}{8}+\frac{\left|f^{\prime}(b)\right|^{q}}{8}\right)^{\frac{1}{q}}\right. \\
& \left.+\left(T_{16}\left(a, b, \frac{2 a b}{a+b}\right)\right)^{\frac{1}{p}}\left(\frac{\left|f^{\prime}(a)\right|^{q}}{8}+\frac{3\left|f^{\prime}(b)\right|^{q}}{8}\right)^{\frac{1}{q}}\right] .
\end{aligned}
$$

\section{Competing interests}

The authors declare that they have no competing interests.

\section{Authors' contributions}

All authors have contributed to all parts of the article. All authors read and approved the final manuscript.

\section{References}

[1] M. Alomari, M. Darus, Some Ostrowski's type inequalities for convex functions with applications, RGMIA Res.Rep. Collect., 13(1) (2010), Article 3, 1-14.

[2] S. S. Dragomir, T. M. Rassias, Ostrowski type inequalities and applications in numerical integration, Kluwer Academic Publishers, 2002.

[3] İ. İşcan, Hermite-Hadamard type inequalities for harmonically convex functions, Hacet. J. Math. Stat., 43 (6) (2014), $935-942$.

[4] İ. İşcan, Ostrowski type inequalities for harmonically s-convex functions, Konuralp Jurnal of Mathematics, Volume 3, No 1 (2015) 63-74.

[5] İ. İşcan, S. Numan, Ostrowski type inequalities for harmonically quasi-convex functions, Elect. J. Math. Anal. App., 2(2) (2014) 189-198.

[6] A. A. Kilbas, H. M. Srivastava, J. J. Trujillo, Theory and Applications of Fractional Differential Equations, Elsevier, Amsterdam, 2006

[7] A. Ostrowski, Über die Absolutabweichung einer differentienbaren Funktionen von ihren Integralmittelwert. Comment. Math. Hel, 10 (1938), 226-227.

[8] M. E. Özdemir, Ç. Yıldız, New Ostrowski Type Inequalities for Geometrically Convex Functions, Int. J. Modern Math. Sci., 8(1) (2013) 27-35.

[9] E. Set, M. E. Özdemir, M. Z. Sarıkaya, New inequalities of Ostrowski's type for $s$-convex functions in the second sense with applications, Facta Uni. Ser. Math. Inform. 27(1) (2012) 67-82. 